\title{
ARTICLE
}

Molecular Diagnostics

\section{Transcription factors WT1 and p53 combined: a prognostic biomarker in ovarian cancer}

\author{
Julia H. Carter ${ }^{1}$, James A. Deddens ${ }^{2}$, Gretchen Mueller ${ }^{1}$, Thomas G. Lewis ${ }^{1}$, Mariah K. Dooley ${ }^{1}$, Michelle C. Robillard ${ }^{1}$, Molly Frydl ${ }^{1}$, \\ Lydia Duvall ${ }^{1}$, Jackson O. Pemberton ${ }^{3}$ and Larry E. Douglass ${ }^{1}$
}

BACKGROUND: New approaches to ovarian cancer are needed to improve survival. Wilms' tumour 1 (WT1) is a tumour-associated antigen expressed in many ovarian cancers. P53 is also often altered. The clinical significance of the combined expression of these two transcription factors has not been studied.

METHODS: One hundred ninety-six ovarian tumours were classified histopathologically. Tumours were stained for WT1 and p53 immunohistochemically. Stains were analysed according to tumour type, grade and FIGO stage. Kaplan-Meier analyses on 96 invasive carcinomas determined whether categorical variables were related to survival.

RESULTS: WT1 and p53 were related to ovarian tumour type, grade, FIGO stage and patient survival. Uniform nuclear p53 expression was associated with invasion and WT1 expression was associated with advanced grade, FIGO stage and poor survival. When WT1 and p53 were both in the age-adjusted Cox model, WT1 was significant while p53 was not. When we combined tumours expressing WT1 and p53, then adjusted for age and tumour subtype, the hazard ratio compared to tumours without WT1 and with normal p53 was 2.70; when adjusted for age and FIGO stage, the hazard ratio was 2.40.

CONCLUSIONS: WT1, an antigen target, is a biomarker for poor prognosis, particularly when combined with altered p53.

British Journal of Cancer (2018) 119:462-470; https://doi.org/10.1038/s41416-018-0191-x

\section{INTRODUCTION}

Epithelial ovarian cancer (OvCa) is the leading cause of death from gynaecologic cancers and has the highest mortality rate of any female reproductive cancer. In 2012, worldwide incidence of ovarian cancer was 239,000 new cases, with 152,000 ovarian cancer deaths. ${ }^{1}$ Most patients are diagnosed at late stages and have a poor prognosis. Radical surgery and platinum-based chemotherapy are the primary treatment. When relapse occurs, there are few treatment options. The five-year survival rate of women with distant metastases is only $17 \%{ }^{2}$ These statistics highlight the need for new approaches to OvCa therapy.

Epithelial OvCa is a heterogeneous disease with multiple subtypes that arise either from extraovarian tissues such as the fallopian tube, endometrium, endometriosis, the gastrointestinal tract, or in some cases from the ovarian surface epithelium or inclusion cysts arising from this surface epithelium. ${ }^{3,4}$ The morphological subtypes of epithelial OvCa include serous, endometrioid, clear cell and mucinous. Histopathological, molecular and genetic studies suggest that these tumours can be divided into two broad categories: type 1, which are indolent, can develop from precursor lesions such as borderline tumours, and are usually confined to the ovary at diagnosis; and type 2, which can arise de novo from the tubal or ovarian surface epithelium, are genetically unstable, rapidly progress and are often diagnosed at late stages. ${ }^{5}$ Type 1 tumours include low-grade serous and endometrioid, mucinous and clear cell carcinomas. Type 2 tumours include high-grade serous and endometrioid carcinomas, undifferentiated carcinomas and carcinosarcomas. Degree of differentiation determines grade with borderline tumours deviating minimally from benign tissues, grade 1 tumours being well differentiated, grade 2 tumours being moderately differentiated and grade 3 tumours being poorly differentiated. Surgical staging of OvCa is by the International Federation of Gynaecology and Obstetrics (FIGO) classification: stage I tumour is localised to one or both ovaries; stage II tumour is associated with pelvic extension; stage III tumour has spread into the abdominal cavity or to retroperitoneal lymph nodes; stage IV tumour is present in liver parenchyma or distant metastases. ${ }^{6}$ Although tumour subtype, grade and stage are related to survival, none offer specific targets for therapy.

Immunotherapy is a treatment modality that could be efficacious in OvCa. OvCa is associated with an immune response in many patients. ${ }^{7-11}$ Therapeutic vaccines are the most studied immunotherapeutic strategy in epithelial OvCa. ${ }^{11,12}$ Any mutant, overexpressed or abnormally expressed protein in cancer cells can be a target for cancer vaccines and/or T cell therapy. ${ }^{13}$ Missense mutations in TP53 are common in OvCa and, due to gain of function, can harbour a poor prognosis in some, but not all, studies. ${ }^{3,14-16}$ Similarly, the Wilms' tumour protein (WT1) is expressed in many OvCas and is a poor prognostic factor. ${ }^{17,18}$ Both WT1 and p53 are tumour-associated antigens. ${ }^{13}$ WT1 is

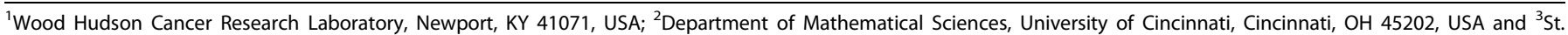
Elizabeth Healthcare, Edgewood, KY 41018, USA

Correspondence: Julia H. Carter (jcarter@woodhudson.org)

Received: 5 May 2018 Revised: 22 June 2018 Accepted: 25 June 2018

Published online: 30 July 2018 
ranked first in pilot prioritisation out of 75 cancer antigens based on predefined criteria, including therapeutic efficacy and immunogenicity. ${ }^{13}$ Molecular studies in multiple models indicate that these two transcription factors, p53 and WT1, interact both physically and functionally. ${ }^{19-23}$ However, the clinical significance of p53 and WT1 expression combined has not been studied in OvCa and hence the potential for the combined expression to be a clinically useful prognostic factor and a predictive marker for response to immunotherapy is not known.

Here we report that the combination of these two immunohistochemically (IHC) detected transcription factors and tumour-associated antigens diffusely expressed in some OvCas, varies both within and between morphologic subtypes of ovarian tumours and that IHC detection of these tumourassociated antigens in OvCa could be useful biomarkers allowing better prognostication and patient selection for newer immunologic approaches targeting WT1 for therapy of lethal OvCa.

\section{MATERIALS AND METHODS}

Tissues studied

Archived, formalin fixed, paraffin-embedded (FFPE) tissues were donated to Wood Hudson Cancer Research Laboratory by St. Elizabeth Healthcare in Northern Kentucky and are preserved in the environmentally controlled Wood Hudson Biospecimen Repository. The study had the approval of the Institutional Review Board of St. Elizabeth Healthcare. Wood Hudson Cancer Research Laboratory has an HIPAA waiver from St. Elizabeth Healthcare. In addition to the tissues from St. Elizabeth Healthcare, FFPE tissues were obtained from the Cooperative Human Tissue Network, Birmingham, Alabama.

A total of 196 archived FFPE surgical specimens obtained from surgeries performed between 1981 and 2006 were stained and evaluated. Ovarian tumours were classified according to subtype by a Board Certified pathologist (LED). For purposes of analysis, ovarian tumours were grouped according to the classification system proposed by Kurman and Shih $^{4,5}$ (Table $1 \mathrm{~A}$ and Fig. 1a). Type 1 tumours (total, $N=51)$ were low-grade serous $(N=9)$, lowgrade endometrioid $(N=6)$, low-grade mixed epithelial $(N=4)$, clear cell $(N=14)$ and mucinous carcinomas $(N=18)$. Type 2 tumours $(N=96)$ were high-grade serous $(N=64)$, high-grade endometrioid $(N=25)$, high-grade mixed epithelial $(N=4)$ and carcinosarcoma $(N=3)$. In addition, 49 borderline ovarian tumours were studied including serous borderline tumours $(N=28)$, mucinous borderline tumours $(N=20)$ and one mixed epithelial borderline tumour.

Clinical follow-up was obtained with the assistance of Tumour Registrars at St. Elizabeth Healthcare from The Kentucky Cancer Registry (part of the SEER network) for 134 of the 136 patients with tissues donated by St. Elizabeth Healthcare; two patients were lost to follow-up. Most patients with type 2 and type 1 tumours received further treatment ( $87 \%$ and $65 \%$, respectively) while patients with borderline tumours rarely received further treatment (7\%). Follow-up information was available for up to 25 years (median 3.9 years). For type 1 patients $(N=35)$, follow-up time ranged from 0 to 25 with a median of 8.84 , for type 2 patients $(N=61)$ the range was also $0-25$ with a median of 2.67. Seventyfive percent of patients with type $2 \mathrm{OvCa}$ in this study died from ovarian cancer while $40 \%$ of type 1 patients died from OvCa.

Clinical follow-up was not available for the 59 patients with tumours obtained from the Cooperative Human Tissue Network. Since only two patients with borderline ovarian tumours died from their disease, all survival analyses were on 96 patients with type 1 and type 2 OvCas with follow-up data.

Immunohistochemistry

Air-dried 5 micron paraffin sections were dewaxed in Clearite, and rehydrated through a graded series of alcohols to distilled water. They were placed in tris-buffered saline (TBS) containing $0.05 \%$ Tween. Following rehydration, antigen retrieval was with $1 \times$ Dako Antigen Retrieval Solution, pH 9.0 (Dako, Carpinteria, CA, USA; cat. no.: S2367), heated to $97-98^{\circ} \mathrm{C}$ in a water bath for $20 \mathrm{~min}$. Sections were allowed to cool for $20 \mathrm{~min}$, and then rinsed in deionised water and placed in TBS-Tween. Adjacent sections were stained immunohistochemically for WT1, p53 or lgG negative controls. WT1 was detected with a mouse monoclonal antibody clone 6F-H2 against the $\mathrm{N}$ terminus amino acids, $1-181$ (Dako; cat. no.: M3561, $1.706 \mathrm{microg} / \mathrm{mL}$ ) or mouse lgG1 control (Dako; cat. no.: X0931, $1.706 \mathrm{microg} / \mathrm{mL}$ ). p53 expression was detected with a mouse monoclonal antibody, clone DO-7 (Dako; cat. no.: M7001, $0.137 \mathrm{microg} / \mathrm{mL}$ ) or a mouse lgG2B control (Dako; cat. no.: X0944, $0.137 \mathrm{microg} / \mathrm{mL}$ ). Others have reported that this antibody is suitable for detecting all p53 variants identified by massive parallel sequencing. ${ }^{16}$ Both antibodies and their lgG controls were diluted with Dako diluent (cat. no.: S0809). Sections were stained for 60 min using a Dako autostainer and the LSAB2 staining system (Dako) with diaminobenzidine as chromagen. Immunohistochemically stained sections were counterstained with methyl green, dehydrated in butanol and Clearite, and coverslipped with

Table 1. Patient characteristics (subtype, FIGO stage, and tumour grade are not $100 \%$ correlated with death from OvCa)

\begin{tabular}{|c|c|c|c|c|c|c|}
\hline \multicolumn{7}{|c|}{ A. All patients $(N=196)$} \\
\hline \multicolumn{7}{|c|}{$\begin{array}{l}\text { Tumour } \\
\text { subtype }\end{array}$} \\
\hline \multirow{3}{*}{$\begin{array}{l}\text { Type } 2 \\
\text { Type } 1 \\
\text { Borderline }\end{array}$} & 96 & \multirow{2}{*}{$\begin{array}{l}64.0 \pm 1.4 \\
58.0 \pm 1.9\end{array}$} & \multirow{3}{*}{$\begin{array}{l}28(29.2 \%) \\
34(66.7 \%) \\
40^{\mathrm{a}}(81.6 \%)\end{array}$} & \multirow{3}{*}{$\begin{array}{l}68(70.2 \%) \\
17(33.3 \%) \\
9^{a}(18.4 \%)\end{array}$} & \multirow{3}{*}{$\begin{array}{l}0 \\
45(88.2 \%) \\
n / a\end{array}$} & \multirow{3}{*}{$\begin{array}{l}96(100 \%) \\
6(11.8 \%) \\
n / a\end{array}$} \\
\hline & 51 & & & & & \\
\hline & 49 & $49.2 \pm 2.4$ & & & & \\
\hline
\end{tabular}

\begin{tabular}{|c|c|c|c|c|c|c|c|}
\hline \multicolumn{8}{|c|}{ B. Patients with clinical follow-up $(N=137)$} \\
\hline $\begin{array}{l}\text { Tumour } \\
\text { subtype }\end{array}$ & $N$ & $\begin{array}{l}\text { Average age } \\
\text { (+/-SEM) }\end{array}$ & $\begin{array}{l}\text { FIGO stage I, } \\
\text { II } n(\%)\end{array}$ & $\begin{array}{l}\text { FIGO stage III, } \\
\text { IV } n(\%)\end{array}$ & $\begin{array}{l}\text { Tumour grade 1, } \\
2 n(\%)\end{array}$ & $\begin{array}{l}\text { Tumour grade } \\
3 n(\%)\end{array}$ & $\begin{array}{l}\text { Died OvCa } \\
n(\%)\end{array}$ \\
\hline Type 1 & 35 & $58.1 \pm 2.5$ & $29(82.8 \%)$ & $6(17.1 \%)$ & $32(91.4 \%)$ & $3(8.6 \%)$ & $13(39.4 \%)$ \\
\hline Borderline & 41 & $50.0 \pm 2.7$ & $36^{\mathrm{a}}(87.8 \%)$ & $5^{\mathrm{a}}(12.2 \%)$ & $\mathrm{n} / \mathrm{a}$ & $\mathrm{n} / \mathrm{a}$ & $2(5 \%)$ \\
\hline
\end{tabular}

Only patients with type 2 and type 1 ovarian cancers $(N=96)$ were used in survival analyses. ${ }^{a}$ Implants not metastases 


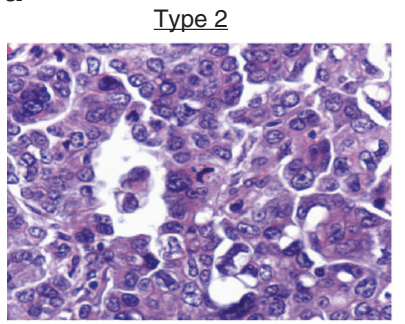

Serous carcinoma

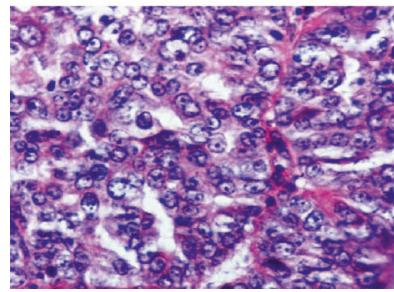

Endometrioid carcinoma

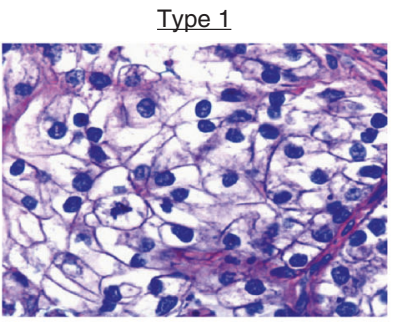

Clear cell carcinoma

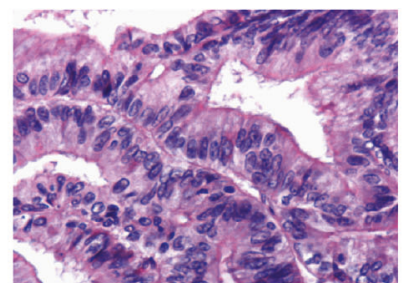

Mucinous carcinoma

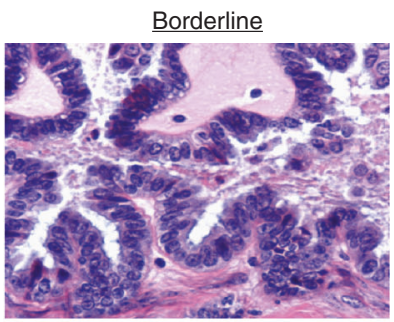

Serous borderline

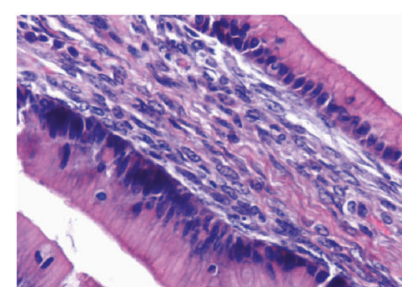

Mucinous borderline

b

Kaplan-Meier survival analysis: survival depends upon tumour type, grade and FIGO stage
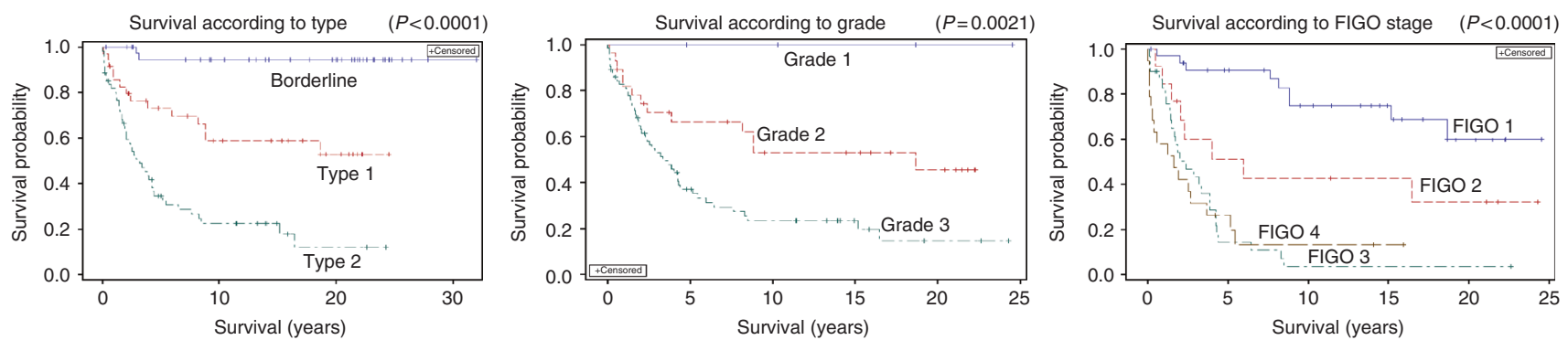

Fig. 1 a Histopathology of ovarian tumour subtypes $(\times 400)$ : Serous carcinoma. Most frequent ovarian carcinoma. It can be either high grade (type 2) or low grade (type 1). Endometrioid carcinoma. Can be either high grade (type 2) with few glandular spaces, or low grade (type 1) with glandular spaces suggestive of endometrial glands. Clear cell carcinoma. This type 1 carcinoma contains cells with clear cytoplasm. Mucinous carcinoma. Mucin containing cells in this type 1 carcinoma are arranged in a disorderly fashion. Serous borderline. Tumour cells contain eosinophilic or amphophilic cytoplasm and are columnar or cuboidal in shape and line a cystic cavity in an orderly fashion. Mucinous borderline. Tumour cells are orderly in their arrangement, line a cystic cavity, and contain much mucin, which maybe either eosinophilic or basophilic. b Kaplan-Meier survival analysis demonstrates that patient survival depends upon ovarian tumour type, grade, and FIGO stage $(p<0.0001)$

Permount (Fisher Healthcare, Hanover Park, IL, USA). A slide of fallopian tube from a single case was included in every $\mathrm{IHC}$ run as a staining control along with a buffer control.

Immunohistochemically stained sections were evaluated by two observers (L.E.D. and J.H.C.) using a Nikon Coolscope (Nikon Instruments, New York, NY, USA). Tumours were considered positive for WT1 if $75-100 \%$ of their nuclei were stained intensely $(3+)$ by the WT1 antibody (Histoscore $=$ Area $\times$ Intensity $=2.7$ ). Tumours were considered to have altered p53 expression if either $75-100 \%$ of their nuclei were stained strongly (2.5-3 + intensity; Histoscore $=2.25-2.7$ ) by the p53 antibody or if the tumour was totally negative. ${ }^{16,24,25}$ Tumours were considered to have normal p53 expression if there were variably stained scattered positive nuclei in the tumour (more than $10 \%$ but less than $75 \%$ of nuclei). Observers were blinded to patient outcome.

\section{Study design}

This was a retrospective study in which patients with ovarian tumours were selected from tumour registries without knowledge of clinical outcome. Although surgical pathology reports were obtained for each case, a single pathologist (L.E.D.) reviewed, diagnosed and graded an H\&E-stained slide of each specimen studied. Clinical information obtained as part of the study included patient age, date of diagnosis, stage of disease, date of recurrence, site of metastases, if patient had additional forms of cancer, date of death (or date known to be alive) and if death was due to OvCa. Variables analysed were subtype of OvCa, grade of tumour, stage of disease, nuclear WT1 expression, normal and altered p53 expression, and survival.

Statistical analysis methods

A total of 196 archived FFPE surgical specimens of ovarian tumours were classified histopathologically. Tumours were stained for expression of WT1 and p53 immunohistochemically. Stain patterns were analysed according to tumour type, grade and FIGO stage. Kaplan-Meier analyses (log-rank tests) were conducted on 96 patients with type 1 and type 2 OvCa with follow-up data, to determine whether categorical variables were related to survival and to obtain estimates of survival curves. Cox proportional hazards regression models were conducted to determine hazard ratios of categorical variables as well as to determine interactions and to adjust for possible confounding variables (e.g., age).

\section{RESULTS}

Altered p53 expression is related to ovarian tumour morphology Table 2A gives the p53 immunohistochemical staining patterns of the 196 tumours in our data set according to morphologic cell 


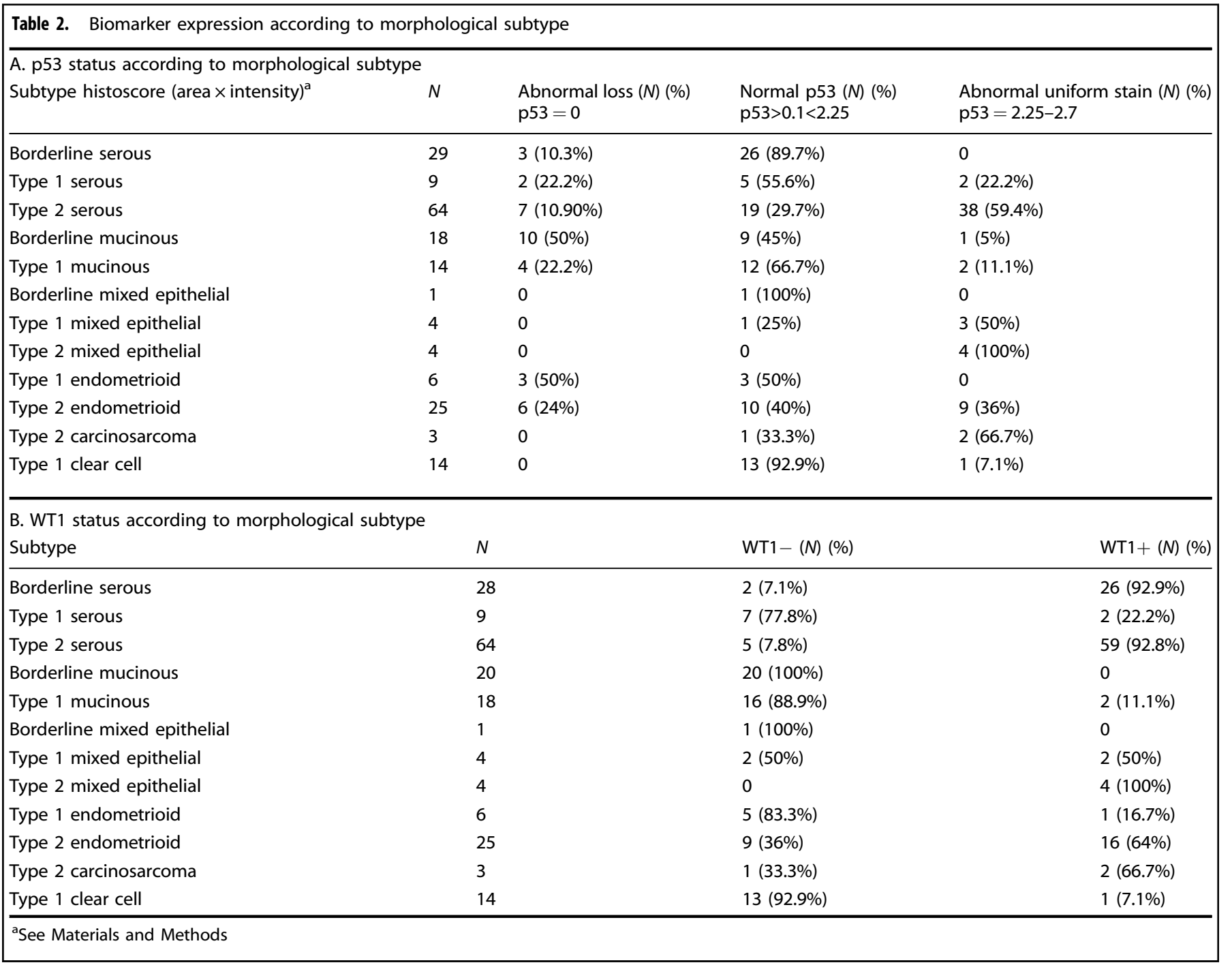

type. The normal p53 stain pattern (variable stain in $10-75 \%$ nuclei) was found in nearly all clear cell OvCa and borderline serous tumours, and also in $31-67 \%$ of borderline mucinous tumours, and serous, mucinous and endometrioid carcinomas and carcinosarcoma subtypes (Table 2A). An absence of p53 immunoreactivity was found in $50 \%$ and $22 \%$, respectively, of borderline mucinous tumours and mucinous OvCa. Only 10-12\% of borderline serous tumours and invasive serous carcinomas and $29 \%$ of endometriod OvCa were without p53 immunoreactivity. In contrast, $22-67 \%$ of serous, mixed epithelial, endometriod and carcinosarcomas had near uniform nuclear immunostaining for p53. Notably, near uniform nuclear immunostaining for p53 was found only in invasive OvCa and was not found in borderline serous tumours.

Nuclear expression of WT1 is related to ovarian tumour morphology

WT1 expression according to ovarian tumour morphology is given in Table 2B. The known association of nuclear WT1 expression with serous differentiation in the ovary was found in both borderline serous ovarian tumours and type 1 and type 2 serous OvCa. Almost all of 64 high-grade serous carcinomas expressed WT1 in $75-100 \%$ of their nuclei. OvCa with other morphologic differentiation also expressed WT1. Approximately half of ovarian endometrioid carcinomas expressed WT1 as well as most mixed epithelial cell OvCa and most carcinosarcomas. Two mucinous and one clear cell OvCa also expressed nuclear WT1. Although serous borderline tumours did not express uniform p53 stain, all serous borderline tumours, which are non-invasive and are minimally deviated histologically from normal ovarian tissues, expressed nuclear WT1.

Altered p53 and nuclear WT1 expression combined are related to tumour type, grade and stage

Four combinations of p53 IHC stain pattern and nuclear WT1 expression were found in OvCa: normal p53 stain pattern and no nuclear WT1 expression ( $\left.\mathrm{p} 53 / \mathrm{WT} 1^{-}\right)$; altered $\mathrm{p} 53$ stain pattern and no nuclear WT1 expression ( $553^{\text {alt }} / \mathrm{WT} 1-$ ); normal $\mathrm{p} 53$ stain pattern and nuclear WT1 expression $\left(\mathrm{p} 53 / \mathrm{WT} 1^{+}\right)$; and altered $\mathrm{p} 53$ IHC stain pattern and nuclear WT1 expression (p53 alt $/ \mathrm{WT}^{+}$) (Fig. 2). Taken together, these four combinations of IHC detected p53 and WT1 nuclear expression were related to ovarian tumour type, ovarian tumour stage and ovarian tumour grade (Table 3A, B, C).

Data in Table 3A show that the percentages of type 1 and type 2 tumours with the four combinations of IHC detected p53 and WT1 nuclear expression differed significantly $(p<0.0001)$. These data suggested that the difference between type 2 and type 1 tumours is 
Normal p53/WT1 negative

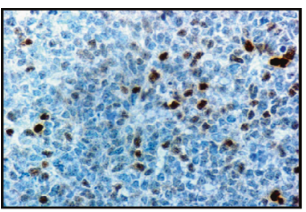

Normal p53

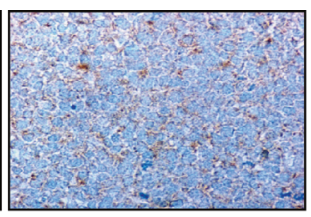

WT1 negative

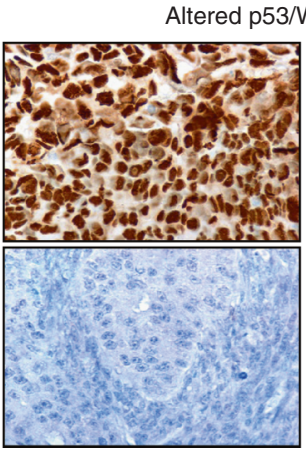

Altered p53

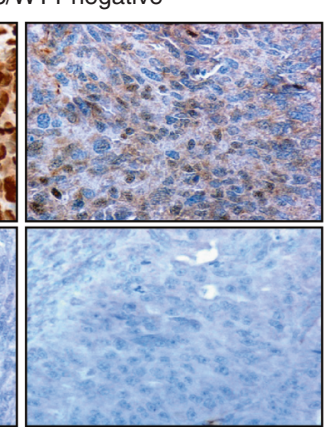

WT1 negative
Normal p53/WT1 positive

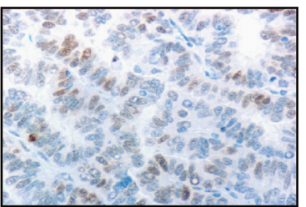

Normal p53

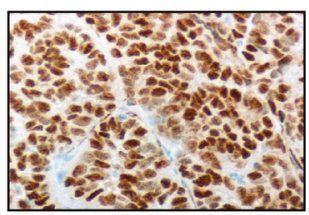

WT1 positive

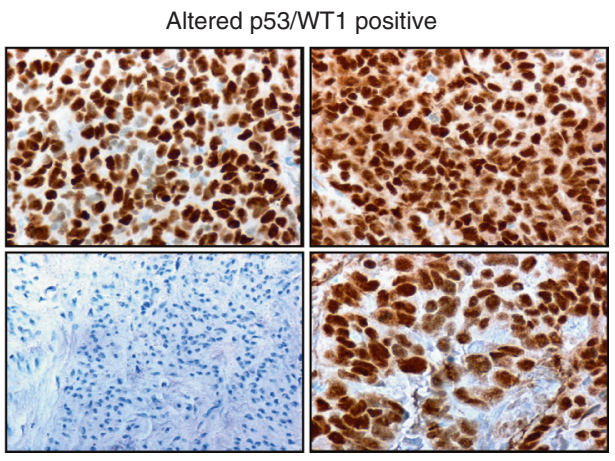

Altered p53

WT1 positive

Fig. 2 Combinations of WT1 and p53 expression in invasive OvCa $(\times 400)$. Expression of p53 was considered "Normal" when nuclei were variably stained and scattered positive nuclei were seen in more than $10 \%$ but less than $75 \%$ of nuclei (nuclear histoscore area $\times$ intensity p 53 $>0.1<2.25$ ). p53 expression was considered "Altered" when tumour cells were strongly positive $2^{+}-3^{+}$in $75-100 \%$ nuclei (abnormal uniform stain, upper image, nuclear histoscore area $\times$ intensity $\mathrm{p} 53=2.25-2.7$ ) or completely devoid of stain (abnormal loss lower image, nuclear histoscore area $\times$ intensity p53 =0). WT1 was considered positive when $75-100 \%$ of nuclei expressed this transcription factor. Images illustrate the four combinations of expression of these two transcription factors and tumour-associated antigens

more highly related to expression of nuclear WT1 by type 2 tumours than to alterations in $\mathrm{p} 53$ nuclear stain. These data also suggested that, although as noted above, alterations in immunohistochemically detected p53 nuclear expression are associated with invasive tumours, these alterations in p53 expression are often associated with changes in WT1 expression in lethal type 2 tumours (see below and Fig. 2). These data give evidence for two pathways to lethal type 2 tumours; nuclear expression of WT1, and combined altered p53 and WT1 nuclear expression.

Data in Table 3B show that the percentages of carcinomas at late stages (FIGO III and IV) and at early stages (FIGO I and II) with the four combinations of expression of p53 and nuclear WT1 differed significantly $(p<0.0001)$. These data suggested that invasive ovarian cancer at early stages (FIGO I and II) are associated with p53 alterations, whereas the progression of ovarian cancer to distant metastases (FIGO III and IV) is associated with tumours having nuclear WT1 expression. These data also suggested that alterations in p53 transcription factor protein expression synergised with nuclear WT1 transcription protein expression in distant metastases clinically.

As seen in Table $3 C$, high-grade (grade 3 ) tumours and lowgrade (grades 1 and 2) tumours differed significantly in the four combinations of expression of p53 and WT1 $(p<0.0001)$. These data (Table 3C) suggested that altered p53 expression is associated with WT1 in increased grade, dedifferentiated, ovarian tumours in women.

Taken together, these data showed that tumours that expressed normal p53 and were WT1 negative predominated in type 1, grades 1 and 2, and FIGO I and II OvCa. Tumours with altered p53 expression that were negative for WT1 were found mainly in type 1, grade 2 and FIGO II OvCa. Tumours with normal p53 that expressed WT1 were found mainly in type 2, grade 3 and FIGO III OvCa. Importantly, type 2, grade 3 and FIGO IV OvCa had both altered p53 and WT1 nuclear expression. Overall, OvCa that had both altered p53 transcription factor protein expression and WT1 transcription factor protein expression differed significantly from OvCa that had normal p53 expression and were negative for WT1 $(p<$ 0.0001).
Histopathologic subtypes, tumour grade, FIGO stage of disease and patient age modify survival from ovarian cancer

As suggested by 'REporting recommendations for tumour MARKer prognostic studies(REMARK) ${ }^{26}$ we first studied the 137 ovarian tumours in our data set for standard prognostic variables including histopathologic subtype, tumour grade and stage of disease (Table 1B and Fig. 1b) for comparison to the proposed prognostic biomarkers of survival, namely altered immunohistochemically detected p53 and WT1 nuclear expression. Since only two patients with borderline tumours died of their disease, further survival analyses only considered the 96 patients with type 1 and type 2 OvCa.

Statistical analyses found that patient age was a confounding factor for survival analyses in the 137 tumours (Table 1B). Survival analyses on 96 patients with type 1 or type 2 OvCa (Fig. 1b) showed that OvCa type was significant $(p=0.0003)$. Median survival for patients with type 2 OvCa was 3.21 years while median survival for type 1 OvCa was at least 18.65 years. After adjusting for age, the hazard ratio for patients with type 2 vs. type 1 OvCa was $2.75(p=0.0011)$. Tumour grade was also significant $(p=0.0072)$. Median survival for patients with grade 3 OvCa was 3.35 years while median survival for those with grade 2 OvCa was at least 18.65 years. The hazard ratio for grade 3 vs. grade 2 OvCa was $2.54(p=0.0037)$ after adjusting for age. FIGO stage was significant $(p<0.0001)$. Median survival for patients at FIGO stages IV, III, II and I was 1.64, 2.37, 5.95 and at least 18.65 years, respectively. The hazard ratios for FIGO IV, III, II vs I were $6.32(p<0.0001), 6.62(p<0.0001), 2.45(p=0.0674)$ after adjusting for age. The hazard ratio for FIGO III, IV vs. II, I was $4.70(p<0.0001)$ after adjusting for age. Hazard ratio analysis demonstrated that tumour type and grade were highly associated, and that FIGO stage was more predictive than either tumour type or grade.

p53 alterations are not significant for patient survival in a multivariant model

Patients with invasive OvCa, type 1 or type 2, having any type of IHC detected altered p53 expression, irrespective of morphology, 
Table 3. WT1 and altered p53 in OvCa

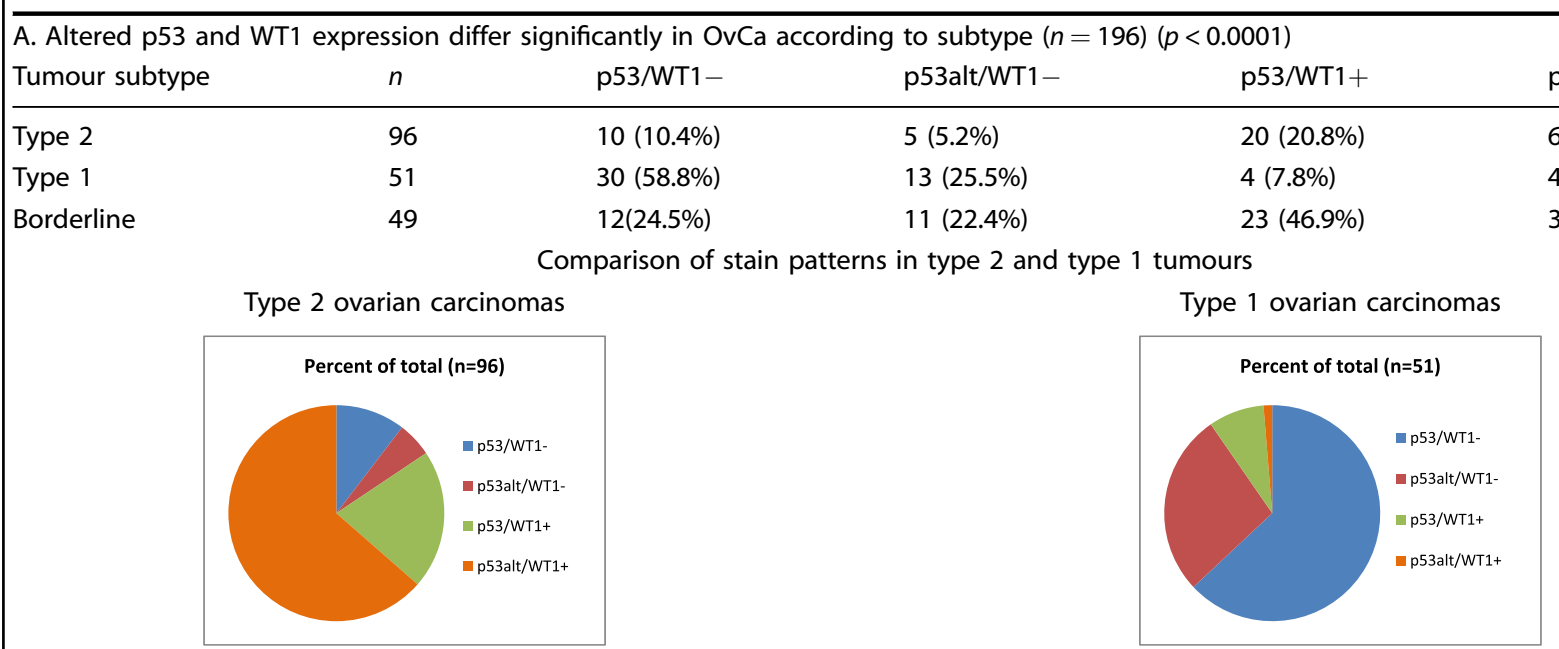

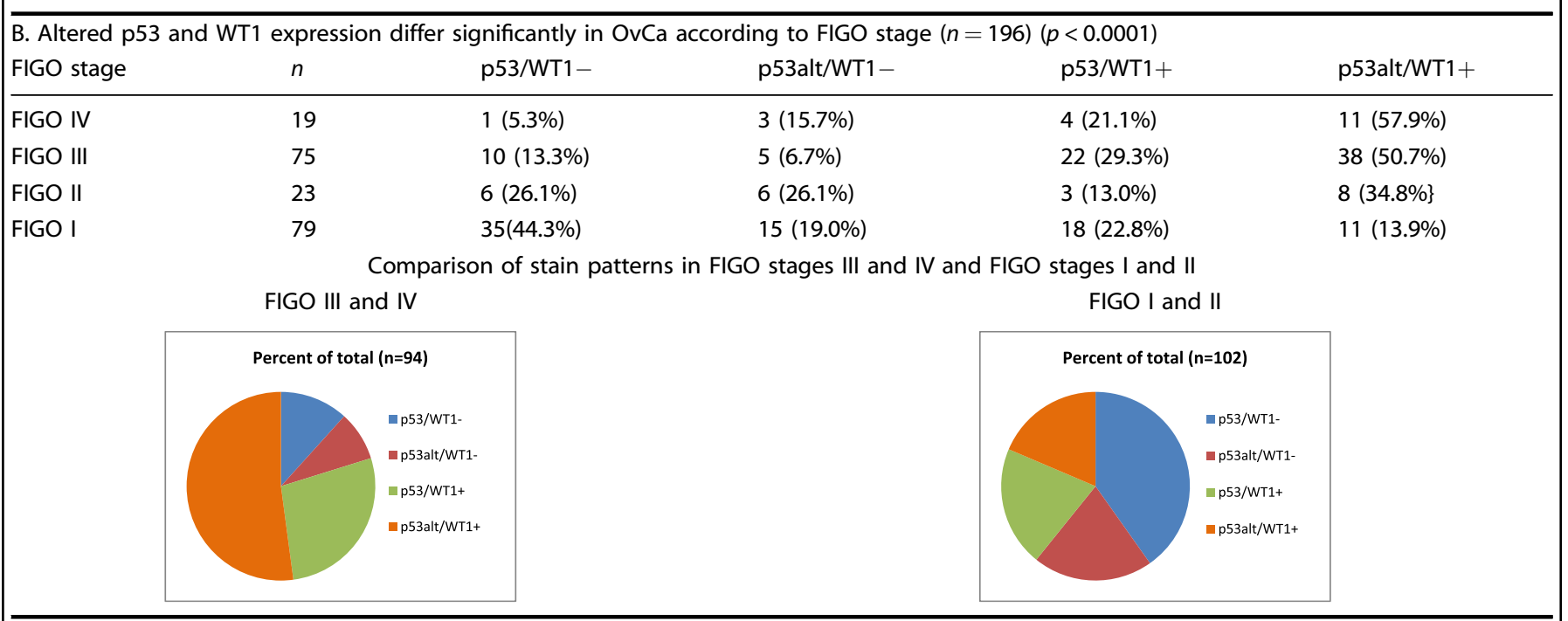

\begin{tabular}{|c|c|c|c|c|c|}
\hline Tumour grade & $n$ & p53/WT1 - & p53alt/WT1 - & p53/WT1+ & p53alt/WT1+ \\
\hline Grade 3 & 102 & $13(12.8 \%)$ & 7 (6.9\%) & $21(20.6 \%)$ & $61(59.8 \%)$ \\
\hline Grade 1 & 5 & $3(60.0 \%)$ & $0(0 \%)$ & $1(20.0 \%)$ & $1(20.0 \%\}$ \\
\hline Borderline/ & 49 & $12(24.5 \%)$ & 11 (22.4\%) & 23 (46.9\%) & 3 (6.2\%) \\
\hline
\end{tabular}

Comparison of stain pattern in high grade vs. low grade ovarian carcinomas

Grade 3 ovarian carcinomas

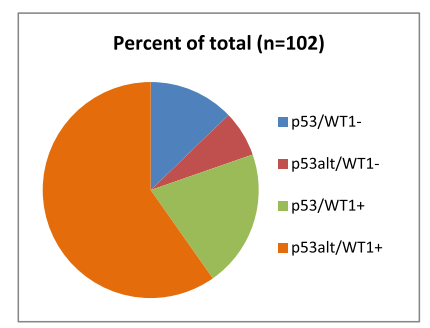

Grade 1 and 2 ovarian carcinomas

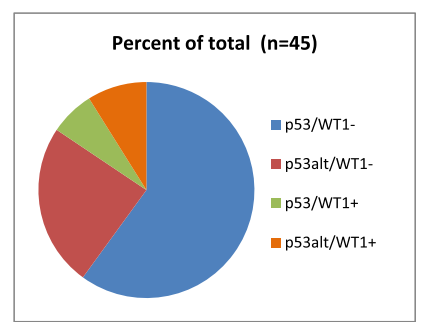

had a shorter survival than those with the wild-type, normal expression pattern ( $p=0.0006$ ) (Fig. 3a). Median survival was 2.30 and at least 18.65 years, respectively. However, after adjusting for age and tumour subtype (type 1 and type 2), the hazard ratio for altered p53 was $1.52(p=1.590)$ and therefore not significant. If we adjust for age and FIGO, the hazard ratio was $1.62, p=0.0902$. The apparent difference in survival between patients with type 1 and type 2 OvCa with the abnormal loss of p53 nuclear stain and 
Table 3 continued

D. Altered p53 and WT1 expression differ significantly in OvCa used in survival analyses according to subtype $(n=96)(p<0.0001)$

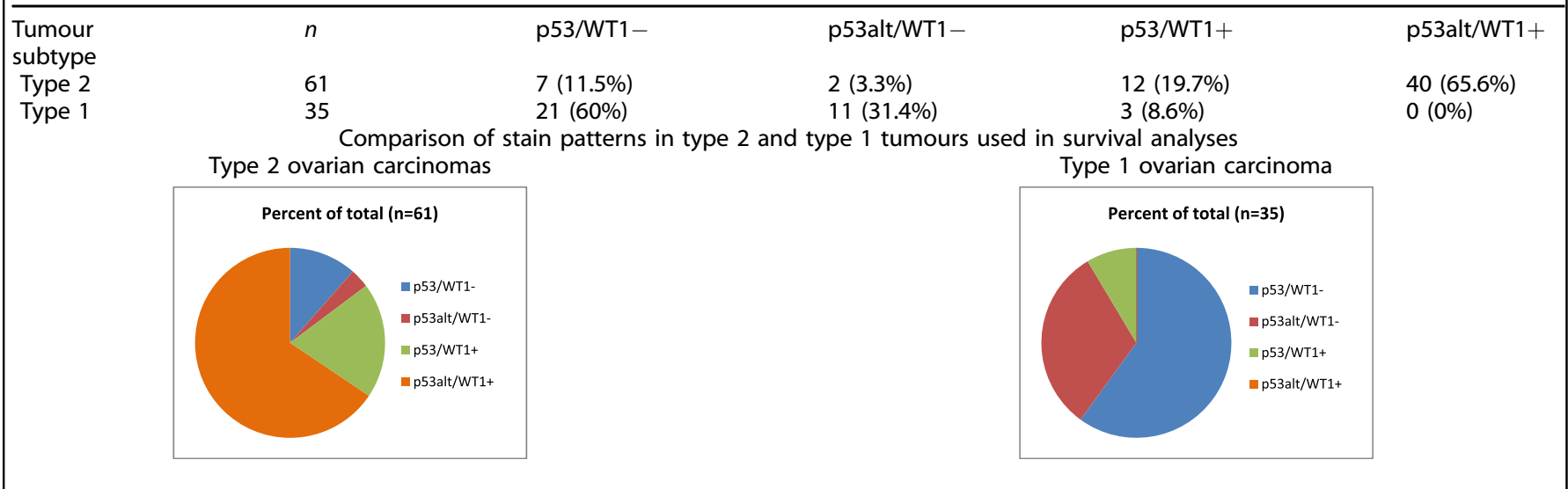

the abnormal uniform nuclear p53 stain did not reach statistical significance ( $p=0.1055$ ) (Fig. $3 b)$.

WT1 expression is a poor prognostic factor for survival of patients with invasive ovarian carcinomas

Although all serous borderline tumours, which are non-invasive and are minimally deviated histologically from normal ovarian tissues, expressed WT1, these borderline tumours were not lethal. In marked contrast, WT1 expression in invasive ovarian carcinomas, irrespective of morphology, was highly associated with poor overall survival of patients $(p<0.0001)$ (Fig. 3c). Median survival was 2.30 and at least 8.84 years, respectively. After adjusting for age and tumour subtype, the hazard ratio for WT1 expression was $2.17(p=0.0589)$. After adjusting for age and FIGO, the hazard ratio was $1.82(p=0.0639)$. When WT1 and p53 were both in the age-adjusted Cox model, WT1 was significant $(p=0.0024)$, while $p 53$ was not $(p=0.2144)$.

Patterns of nuclear WT1 and p53 expression are related not only to ovarian tumour type, grade and FIGO stage, but also to survival Table 3D gives the distribution of the markers in OvCa from the 96 patients used in the survival analysis. The data in Table 3D demonstrate that WT1 and altered p53 expression differed significantly in OvCa according to subtype in patients studied in the survival analyses.

Overall survival from ovarian cancer was highly related to altered p53 and WT1 nuclear expression ( $p<0.0001$, Fig. $3 d$ ). The median survival for patients with OvCa having abnormal p53 nuclear expression and that also expressed nuclear WT1 was 2.30 years. Median survival for patients with OvCa having normal p53 expression that also expressed WT1 was 4.00 years, and was 5.16 years for patients with OvCa having altered p53 nuclear expression but negative for WT. In contrast, median survival for patients with OvCa having normal p53 nuclear expression and without WT1 was 8.84 years. The 20-year survival probability of patients with OvCa without either an altered p53 nuclear stain pattern or nuclear WT1 expression was $67.7 \%$ and was significantly better than other patients (see Fig. 3d). The 20-year survival probability for patients with OvCa negative for WT1 but with altered p53 expression was $35.9 \%$, and was $0 \%$ for patients with OvCa expressing WT1 and the normal p53 stain pattern. Patients with OvCa expressing both nuclear WT1 and altered p53 expression had a 20 -year survival probability of $6.5 \%$. If survival in patients with OvCa having either abnormal p53 nuclear stain pattern or nuclear WT1 expression were combined, then adjusted for age and tumour subtype the hazard ratio compared to patients with OvCa having normal p53 nuclear expression and negative for WT1 was $2.70(p=0.0201)$ and $p=0.0296$ with a hazard ratio of 2.40 when age and FIGO adjusted. We conclude that altered p53 and nuclear WT1 expression combined was associated with reduced survival after adjusting for both age and tumour subtype as well as age and FIGO stage, and that nuclear WT1 expression was a biomarker for lethal OvCa and that OvCas expressing WT1 are more frequently lethal when p53 is also altered.

\section{DISCUSSION}

'Ovarian cancer' is a group of genetically, biochemically and morphologically different diseases. Not all invasive OvCas of any one morphological type are lethal. Patient survival depends upon the well-known prognostic factors of tumour type, tumour grade and FIGO stage. However, none of these prognostic factors offer a target for therapy. Here we report in a cohort of ovarian carcinoma patients with long-term follow-up that altered p53 and WT1 nuclear expression in invasive OvCa is associated with reduced survival after adjusting for both age and FIGO stage. Since both WT1 and p53 are tumour-associated antigens and WT1 is ranked first in pilot prioritisation out of 75 cancer antigens based on predefined criteria including therapeutic efficacy and immunogenicity, ${ }^{13}$ these proteins offer targets for therapy of lethal OvCa. However, in this cohort of OvCa patients with longterm follow-up, abnormal p53 expression detected immunohistochemically does not predict survival based on multivariate analysis.

The WT1 is a transcription factor protein involved in the transcriptional regulation of genes such as growth factors, regulators of the cell cycle as well as apoptosis, and differentiation markers. ${ }^{27}$ WT1 expression is increased during progression of OvCa to metastasis. ${ }^{27,28}$ Silencing of WT1 in OvCa cell lines with siRNA reduces OvCa cell motility and ability to invade 3D collagenrich matrices. ${ }^{28}$ Conversely, forced overexpression of WT1 in an OvCa cell line increased cell invasion in a Boyden chamber assay as well as cell proliferation. ${ }^{27}$

WT1 and p53 are transcription factors and tumour-associated antigens that are differentially co-expressed in all subtypes of OvCa. In multiple experimental models, p53 and WT1 are known to interact physically and functionally. Maheswaran et al. ${ }^{19}$ proposed that under conditions of partial or total p53 tumour functional inactivation, the WT1 protein could be converted from a transcriptional repressor to an activator. Viel et al. $^{29}$ also suggested that when wild-type p53 is scarce or absent, an overproduced WT1 protein could acquire activational properties, thus completely reversing its transcriptional control of growth-related genes. Consistent with these studies, altered p53 expression combined with WT1 expression predominated in type 2, grade 3 and FIGO IV ovarian carcinomas. Moreover, we find that altered p53 expression and WT1 
a

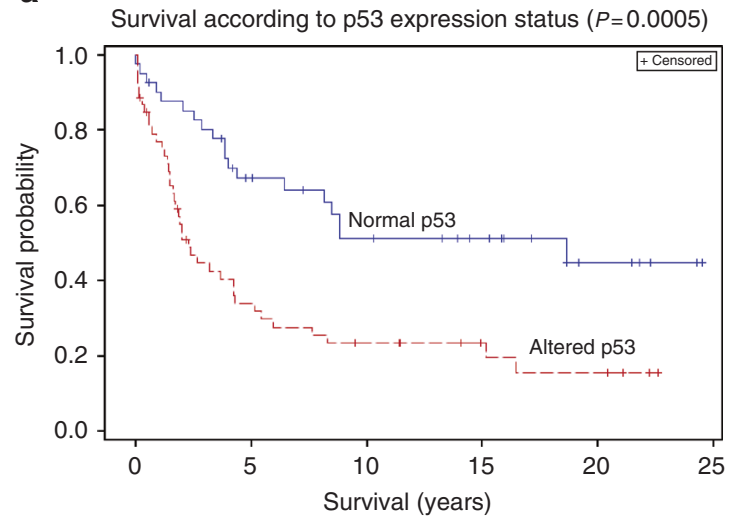

C

Survival according to WT1 expression $(P<0.0001)$

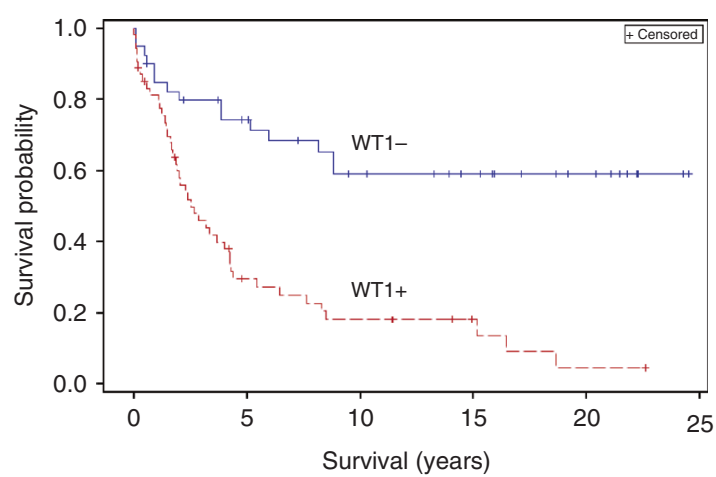

b

Survival according to type of p53 expression $(P=0.0003)$

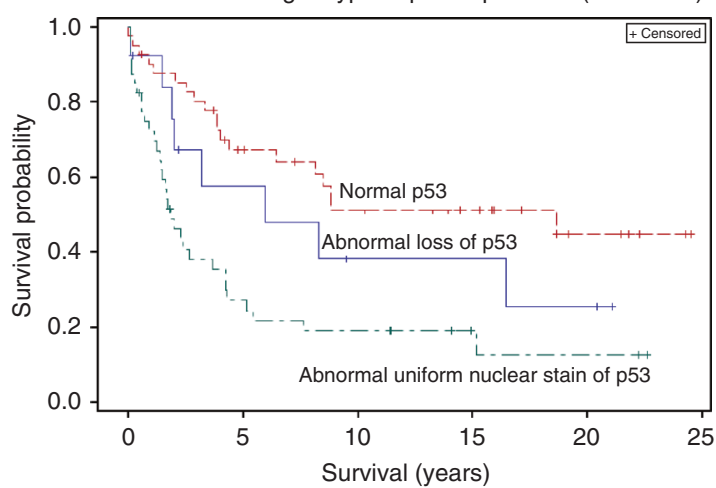

d

Survival according to altered p53 and WT1 $(P<0.0001)$

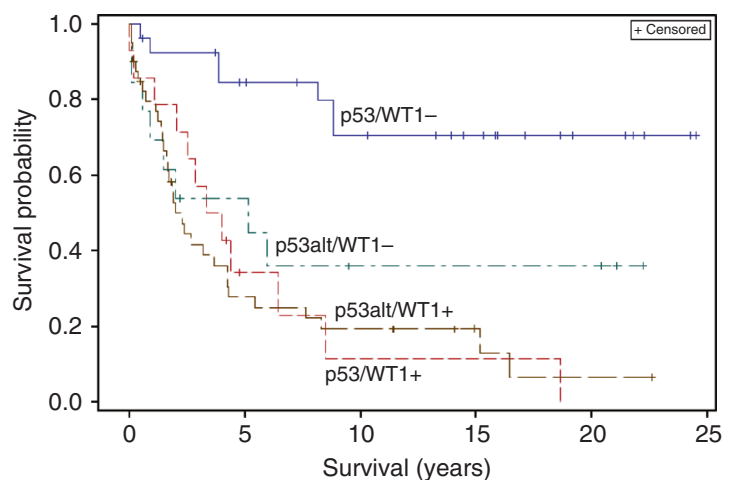

Fig. 3 Clinical relevance of WT1 and altered p53 in OvCa. a Patient survival is highly dependent upon the altered expression of p53 proteins in invasive OvCa (type 1, indolent OvCa, and type 2, aggressive OvCa) $(p=0.0004)$. After adjusting for age and tumour type, the hazard ratio for altered p53 expression is 1.55. b The worse survival in patients with OvCa expressing abnormal uniform p53 nuclear stain vs. abnormal loss of nuclear p53 stain does not reach statistical significance $(p=0.1055)$. c Patient survival is highly dependent upon the expression of Wilms' Tumour 1 (WT1) protein in OvCa (type 1 and type 2$)(p=0.0001)$. After adjusting for age and tumour type, the hazard ratio for WT1 expression is 2.25. $\mathbf{d}$ Co-expression of WT1 and altered p53 increases risk of death from invasive ovarian cancer (hazard ratio $=3.358$ compared to cancers without either protein). Survival is significantly reduced when either WT1 or altered p53 are expressed ( $p=0.0001)$; however, when WT1 is present, the adverse effect of altered p53 expression on survival is increased

expression combined in OvCa is a better predictor of patient survival than tumour type or tumour grade. The effect of ovarian cancer expression of altered p53 and/or WT1 is associated with an increased risk of death after adjusting for both age and FIGO stage when compared to patients with tumours with normal p53 expression and a lack of nuclear WT1 expression (hazard ratio $=2.4$ ).

In contrast to tumour type, grade and FIGO stage, immunohistochemically detected WT1 and altered p53 expression are tumour-associated antigens and transcription factors that, as shown here, identify tumours with an aggressive phenotype and also provide a potential target for immunotherapy. Identifying tumours with a more aggressive phenotype will lead to a rational selection of patients for immunotherapy.

The hazard ratio for death in patients with tumours having WT1 expression but without altered p53 or with both WT1 and altered p53 expression was 3.252 and 3.358, respectively, compared to patients with tumours without either WT1 or altered p53 expression. Vermeij et al. $^{30}$ demonstrated that WT1 overexpression in ovarian tumours was associated with intratumoural Treg and cytotoxic $\mathrm{T}$ cell infiltration, which was an independent prognostic factor for progression-free survival.

Clinical trials of WT1 immunotherapy suggested that WT1 is a strong tumour-associated antigen that can by itself induce some immunological and oncological changes. ${ }^{31,32}$ However, additional approaches such as combined vaccination with other tumour-associated antigens, or standard chemotherapy, or antibodies against immune checkpoints will be needed to overcome the immunosuppressive factors in the ovarian cancer microenvironment. ${ }^{32-34}$ The hope is that a combination of Treg targeting, along with the activation of tumour-specific effector $T$ cells by cancer vaccination or immune checkpoint blockade, will make cancer immunotherapy more efficacious. ${ }^{34}$ As recently reviewed, ${ }^{35}$ response to immune checkpoint blockade depends not only on the molecular characteristics of the tumour, but also on the tumour microenvironment, the immune competence of the patient and environmental influences such as the patient's gut microbiome.

Our data are consistent with the conclusions that: (1) invasive ovarian cancers at early stages (FIGO I and II) are associated with altered nuclear p53 expression, whereas metastatic ovarian cancers (FIGO III and IV) are associated with nuclear WT1 expression; (2) compared to patients without either WT1 or altered p53 expression, patients with tumours expressing altered p53 or nuclear WT1 have decreased survival even when these data are adjusted for age and tumour type or age and FIGO stage; and (3) the transcription factor(s) WT1, or WT1 and altered p53 expression are prognostic biomarkers of poor survival and could be useful predictive biomarkers for stratifying patients eligible for new immunologic approaches targeting the tumour-associated antigen WT1 for therapy of lethal ovarian cancers. 


\section{ACKNOWLEDGEMENTS}

We thank Denise Lucas, HT, Histology Lab Manager and Elyse Hawkins, Wood Hudson Cancer Research Laboratory Biospecimen Repository Manager for their expert assistance. We thank the Department of Pathology of St. Elizabeth Healthcare, as well as Cathy Reising and the Tumour Registry of St. Elizabeth Healthcare for their assistance. We also thank the Cooperative Human Tissue Network, Birmingham, Alabama.

\section{AUTHOR CONTRIBUTIONS}

G.M., T.L., M.D., M.R., M.F., and L.D. acquired data for the work and performed the IHC. L.D. and J.P. acquired data for the work and performed the histopathological diagnoses. L.D. and J.C. performed the semi-quantitative IHC evaluations. J.D. performed the statistical analyses. J.C., J.D., and L.D. wrote the manuscript. J.C. coordinated the study and obtained the funding.

\section{ADDITIONAL INFORMATION}

Ethics approval: This study had the approval of the Institutional Review Board of St. Elizabeth Healthcare. Wood Hudson Cancer Research Laboratory has an HIPAA waiver from St. Elizabeth Healthcare.

\section{Consent for publication: All authors consent for publication of this manuscript.}

Competing interests: The authors declare no competing interests.

Availability of data: The data generated during this study are available from the corresponding author on reasonable request.

Funding: This work was financially supported by grants from the Ovarian Cancer Alliance of Greater Cincinnati, the R. C. Durr Foundation, and the Carol Ann \& Ralph V. Haile, Jr./U.S. Bank Foundation and gifts to the Wood Hudson Cancer Research Laboratory Memorial Fund.

\section{REFERENCES}

1. Prat, J. \& Franceschi, S. World Cancer Report. (eds Stewart, B. W. \& Wild, C. P.) 465-481 (International Agency for Research on Cancer, Lyon, France, 2014).

2. Ovarian Cancer Research Fund Alliance. https://ocrfa.org/patients/about-ovariancancer/treatment/staging-and-grading/stage-4/. (2018).

3. Le Page, C., Huntsman, D. G., Provencher, D. M. \& MMes-asson, A. M. Predictive and prognostic protein biomarkers in epithelial ovarian cancer: recommendation for future studies. Cancers 2, 913-954 (2010).

4. Kurman, R. J. \& Shih, L. M. Molecular pathogenesis and extraovarian origin of epithelial ovarian cancer. Shifting the paradigm. Hum. Pathol. 42, 918-931 (2011).

5. Koshiyama, M., Matsumura, N. \& Konishi, I. Recent concepts of ovarian carcinogenesis: type I and type II. BioMed. Res. Internatl. 2014, 934261 (2014).

6. Prat, J. FIGO Committee on Gynecologic Oncology Staging classification for cancer of the ovary, fallopian tube, and peritoneum. Int J. Gynecol. Obstet. 124, 1-5 (2014).

7. Zhang, L. et al. Intratumoral T cells, recurrence, and survival in epithelial ovarian cancer. N. Engl. J. Med. 248, 203-213 (2003).

8. Curiel, T. J. et al. Specific recruitment of regulatory T cells in ovarian carcinoma fosters immune privilege and predicts reduced survival. Nat. Med. 10, 942-949 (2004).

9. Sato, E. et al. Intraepithelial CD8 + tumor-infiltrating lymphocytes and a high CD8 + /regulatory $\mathrm{T}$ cell ratio are associated with favorable prognosis in ovarian cancer. Proc. Natl Acad. Sci. USA 102, 18538-18543 (2005).

10. Preston, C. C. et al. The ratios of $\mathrm{CD}^{+}{ }^{+} \mathrm{T}$ cells to $\mathrm{CD} 4^{+} \mathrm{CD} 25^{+} \mathrm{FOXP3}^{+}$and VOXP3 $\mathrm{T}$ cells correlate with poor clinical outcome in human serous ovarian cancer. PLOS ONE 8, 1-10 (2013).

11. Coukos, G., Tanyi, J. \& Kandalaft, E. Opportunities in immunotherapy of ovarian cancer. Ann. Oncol. 27(Supplement 1), i11-i15 (2016).

12. Chester, C., Dorigo, O., Berek, J. S. \& Kohrt, H. Immunotherapeutic approaches to ovarian cancer treatment. J. Immunother. Cancer 3, 7 (2015)

13. Cheever, M. A. et al. The prioritization of cancer antigens: A National Cancer Institute pilot project for the acceleration of translational research. Clin. Cancer Res. 15, 5323-5337 (2009).

14. de Graeff, P. et al. Modest effect of p53, EGFR and HER-2/neu on prognosis in epithelial ovarian cancer: a meta-analysis. Br. J. Cancer 101, 149-159 (2009).
15. Bernardini, M. Q. et al. Expression signatures of TP53 mutations in serous ovarian cancers. BMC Cancer 10, 237 (2010).

16. Cole, A. J. et al. Assessing mutant p53 in primary high-grade serous ovarian cancer using immunochistochemistry and massively parallel sequencing. Sci. Rep. 6, 26191 (2016).

17. Netinatsunthorn, W., Hanprasertpong, J., Dechsukhum, C., Leetanaporn, R. \& Geater, A. WT1 gene expression as a prognostic marker in advanced serous epithelial ovarian carcinoma: an immunohistochemical study. BMC Cancer 6, 90 (2006).

18. Qi X-w, ZhangF. et al. Wilms' tumor 1 (WT1) expression and prognosis in solid cancer patients: a systematic review and meta-analysis. Sci. Rep. 5, 8924 (2015).

19. Maheswaran, S. et al. Physical and functional interaction between WT1 and p53 proteins. Proc. Natl Acad. Sci. USA 90, 5100-5104 (1993).

20. Maheswaran, S., Englert, C., Bennett, P., Heinrich, G. \& Haber, D. A. The WT1 gene product stabilizes p53 and inhibits p53-mediated apoptosis. Genes Dev. 9, 2143-2156 (1995)

21. Idelman, G., Glaser, T., Roberts, C. T. Jr \& Werner, H. WT1-p53 interactions in insulin-like growth factor-1 receptor gene regulation. J. Biol. Chem. 278, 3474-3482 (2002)

22. Menke, A. L. et al. Genetic interactions between the Wilms' tumor 1 gene and the p53 gene. Cancer Res. 62, 6615-6620 (2002).

23. Massaoka, M. H. et al. A novel cell-penetrating peptide derived from WT1 enhances p53 activity, induces cell senescence and displays antimelanoma activity in xeno- and sungeneic systems. FEBS Open Bio 4, 153-161 (2014).

24. Yemelyanova, A. et al. Immunohistochemical staining patterns of p53 can serve as a surrogate marker for TP53 mutations in ovarian carcinoma: an immunohistochemical and nucleotide sequencing analysis. Mod. Pathol. 24, 1248-1253 (2011).

25. Lee, S. H. et al. Genetic alteration and immunohistochemical staining patterns of ovarian high-grade serous adenocarcinoma with special emphasis on p53 immunostaining pattern. Pathol. Intern. 63, 252-259 (2013).

26. McShane, L. M. et al. Reporting recommendations for tumor MARKer prognostic studies (REMARK). Nat. Clin. Pract. 2, 416-422 (2005).

27. Liu, Z. et al. High levels of Wilms' tumor 1 (WT1) expression were associated with aggressive clinical features in ovarian cancer. Anticancer Res. 34, 2331-2340 (2014).

28. Barbolina, M. V., Adley, B. P., Shea, L. D. \& Stack, M. S. Wilms tumor gene protein 1 is associated with ovarian cancer metastasis and modulates cell invasion. Cancer 112, 1632-1641 (2008).

29. Viel, A. et al. Molecular mechanisms possibly affecting WT1 function in human ovarian tumors. Int. J. Cancer 57, 515-521 (1994).

30. Vermeij, R. et al. Tumor-infiltrating cytotoxic $T$ lymphocytes as independent prognostic factor in epithelial ovarian cancer with Wilms tumor protein 1 overexpression. J. Immunother. 34, 516-523 (2011).

31. Sugiyama, H. Cancer immunotherapy targeting Wilms' tumor gene WT1 product. Expert. Rev. Vaccin. 4, 503-512 (2005)

32. Coosemans, A., Vergote, I. \& Van Gool, S. W. Wilms' tumor gene 1 immunotherapy in pelvic gynecological malignancies. Expert Rev. Clin. Immunol. 10, 705-711 (2014).

33. Lavoue, V. et al. Immunity of human epithelial ovarian carcinoma: the paradigm of immune suppression in cancer. J. Transl. Med. 11, 147 (2013).

34. Tanaka, A. \& Sakaguchi, S. Regulatory T cells in cancer immunotherapy. Cell Res. 27, 109-118 (2017)

35. Cogdill, A., Andrews, M. C. \& Wargo, J. A. Hallmarks of response to immune checkpoint blockade. Br. J. Cancer 117, 1-7 (2017).

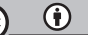

Open Access This article is licensed under a Creative Commons Attribution 4.0 International License, which permits use, sharing, adaptation, distribution and reproduction in any medium or format, as long as you give appropriate credit to the original author(s) and the source, provide a link to the Creative Commons license, and indicate if changes were made. The images or other third party material in this article are included in the article's Creative Commons license, unless indicated otherwise in a credit line to the material. If material is not included in the article's Creative Commons license and your intended use is not permitted by statutory regulation or exceeds the permitted use, you will need to obtain permission directly from the copyright holder. To view a copy of this license, visit http://creativecommons. org/licenses/by/4.0/.

(c) The Author(s) 2018 